\title{
All-cause mortality among people with serious mental illness (SMI), substance use disorders, and depressive disorders in southeast London: a cohort study
}

Chin-Kuo Chang $^{1 *}$, Richard D Hayes ${ }^{1}$, Matthew Broadbent ${ }^{1}$, Andrea C Fernandes ${ }^{1}$, William Lee ${ }^{2}$, Matthew Hotopf ${ }^{2}$, Robert Stewart ${ }^{1}$

\begin{abstract}
Background: Higher mortality has been found for people with serious mental illness (SMI, including schizophrenia, schizoaffective disorders, and bipolar affective disorder) at all age groups. Our aim was to characterize vulnerable groups for excess mortality among people with SMI, substance use disorders, depressive episode, and recurrent depressive disorder.
\end{abstract}

Methods: A case register was developed at the South London and Maudsley National Health Services Foundation Trust (NHS SLAM), accessing full electronic clinical records on over 150,000 mental health service users as a welldefined cohort since 2006. The Case Register Interactive Search (CRIS) system enabled searching and retrieval of anonymised information since 2008. Deaths were identified by regular national tracing returns after 2006. Standardized mortality ratios (SMRs) were calculated for the period 2007 to 2009 using SLAM records for this period and the expected number of deaths from age-specific mortality statistics for the England and Wales population in 2008. Data were stratified by gender, ethnicity, and specific mental disorders.

Results: A total of 31,719 cases, aged 15 years old or more, active between 2007-2009 and with mental disorders of interest prior to 2009 were detected in the SLAM case register. SMRs were 2.15 (95\% Cl: 1.95-2.36) for all SMl with genders combined, 1.89 (1.64-2.17) for women and 2.47 (2.17-2.80) for men. In addition, highest mortality risk was found for substance use disorders ( $\mathrm{SMR}=4.17 ; 95 \% \mathrm{Cl}$ : 3.75-4.64). Age- and gender-standardised mortality ratios by ethnic group revealed huge fluctuations, and SMRs for all disorders diminished in strength with age. The main limitation was the setting of secondary mental health care provider in SLAM.

Conclusions: Substantially higher mortality persists in people with serious mental illness, substance use disorders and depressive disorders. Furthermore, mortality risk differs substantially with age, diagnosis, gender and ethnicity. Further research into specific risk groups is required.

\section{Background}

The potential impact of mental disorders on mortality has been increasingly recognised in recent years. People with serious mental illnesses (SMI, including schizophrenia, schizoaffective disorder, bipolar disorder, and depressive psychosis) live with health disparities, not only

\footnotetext{
* Correspondence: chin-kuo.chang@kcl.ac.uk

'King's College London, Section of Epidemiology, Dept of Health Service and

Population Research, Institute of Psychiatry, London, UK Full list of author information is available at the end of the article
}

resulting from social dysfunction, stigma, and direct consequences of psychopathology, but also potentially from the deleterious physical consequences of long-term antipsychotic use and adverse lifestyle choices (e.g. smoking, diet, illicit drug use, and physical inactivity), particularly contributing to cardiovascular mortality [1-5].

Higher mortality has been found for people with serious mental illness (SMI) in all age groups. In the US, life expectancy for people with SMI from public mental health agencies in eight states for 1997 through 2000 was
C Biomed Central 
reported to be at least $30 \%$ shorter than that for the general population [6]. In the UK, any psychiatric diagnosis was associated with a $65 \%$ higher than expected total mortality in one case register study [7], and a three-fold elevated mortality was found for coronary heart disease in young adults with SMI in a primary care setting, as well as an almost two-fold elevation for those aged 50-75 years, and a more than two-fold increased stroke mortality in all age groups [8]. Furthermore, a greater than 10-fold increased risk of suicide mortality was found among the same groups, itself associated with increased consultation rates, antidepressant prescriptions, and residence in less deprived areas [9].

Despite recent improvements in health care systems, there has been little evidence for benefits on prolonging life expectancy in people with SMI $[5,10,11]$. Instead, a recent systematic review suggested a widening mortality gap over recent decades with the pattern of change suggesting a failure to benefit from population improvements in health rather than an actual increased case fatality rate [11]. Given this persistence of excess mortality, particularly in younger age groups, there is an imperative need for further investigations in this area $[10,12,13]$. Preliminary strategies for preventing premature deaths among people with SMI have been proposed, including the management of suicide risk and physical illness, minimizing polypharmacy, and improving accessibility to physical health care [1]. However, there is limited evidence upon which to base these. Previous analyses of the UK General Practice Research Database (GPRD) have only reported associations between any SMI and mortality and did not differentiate between disorders [8]. Further evidence is required to clarify the characteristics of people with SMI who are at highest risk of mortality.

We therefore investigated excess mortality for people with individual disorders within the SMI grouping, as well as for depressive and substance use disorder diagnoses, drawing on data from the South London and Maudsley NHS Foundation Trust Biomedical Research Centre (SLAM BRC) Case Register, which covers comprehensive secondary mental healthcare provision to a large geographically defined community.

\section{Methods}

\section{Setting and study population}

The SLAM BRC Case Register provides anonymised indepth information derived from electronic medical records relating to secondary mental health care, which includes all specialist care (i.e. apart from that provided by general practitioners) for hospitalization, outpatient care, a broad profile of community care models (both acute care and rehabilitation), psychiatric liaison services to general hospitals, and forensic mental health services.
The protocol for this case register has been described in detail in an open-access publication [14]. SLAM provides comprehensive secondary mental health care to a population of approximately 1.3 million residents of four London boroughs (Lambeth, Southwark, Lewisham and Croydon) as well as tertiary care national referral units. Under the British National Health Service (NHS), all secondary mental healthcare within these four boroughs is provided at no cost to consumers by SLAM, the only exception being people seeking exclusively private healthcare [15]. Electronic clinical records have been used comprehensively across all SLAM services since 2006 and the Case Register Interactive Search (CRIS) system was developed in 2008 to allow searching and retrieval of anonymised information with over 150,000 cases currently represented on the system. CRIS was approved as a dataset for secondary analysis by Oxfordshire Research Ethics Committee C, reference 08/H0606/71.

This analysis focused on recorded mortality over a three-year period from 2007 to 2009. Cases were included if they had had contact with SLAM services (a referral, discharge, or case note entry) from $1^{\text {st }}$ Jan, 2007 to $31^{\text {st }}$ Dec, 2009 and had received an SMI, substance use disorder, depressive episode, or recurrent depressive disorders diagnosis before or during that time. In the duration, routine mortality monitoring was carried out on these patient records, hence patients were "at risk" of death during this period of time. Date of birth and gender were routinely recorded and verified on the SLAM electronic patient electric records in designated fields. Age was calculated at the $1^{\text {st }}$ of July, 2008 (i.e. the mid-point of the "at risk" period). All those who were under the age of 15 at this date were excluded from the analyses.

\section{Mortality and covariates}

NHS number is a unique identifier for UK NHS records, all death certifications are linked to this identifier at a national level, and primary and secondary health service providers are required by law to keep records up to date with respect to this. A list of deceased patients associated with SLAM is downloaded on a monthly basis for the "Service User Death Report" from "the Spine" provided by NHS Care Records Service for whom has been marked as deceased by an update from another patient demographic system. For non-active previous service users, this was taken to be the date of death. Further checking routinely occurs for active service users to corroborate the death. The last monthly download was 31 March, 2010 and completed on 7 April, 2010. Diagnoses recorded in the SLAM BRC Case Register were based on the $10^{\text {th }}$ edition of the World Health Organization International Classification of Diseases (ICD-10). 
In this analysis, patients were classified as having an SMI if they had received at least one of the following diagnoses (identified by corresponding ICD-10 codes) during their time in contact with SLAM services: schizophrenia (F20), schizoaffective disorders (F25), and bipolar affective disorder (F31). Substance use disorders (F10 to F19), depressive episode (F32) and recurrent depressive disorder (F33) were also used for analyses. Separate analyses were carried out for each disorder or grouping i.e. those with more than one primary diagnosis during the follow-up period could appear in more than one 'case' group. Ethnic group classifications applied in the Case Register were: "White British", "Other white background", "East Asian", "South Asian", "African and other black background", "Caribbean", and "Mixed, unknown, and others".

\section{Statistical analysis}

Standardized mortality ratios (SMRs) were calculated by Stata for the three-year observation period, using the number of deaths observed in SLAM records in these three years as the numerator. The denominator was the expected number of deaths in a year estimated by age- and/or gender-specific mortality statistics for the England and Wales population in 2008 multiplied by three [16]. SMRs were calculated using age strata (namely, 15-19, 20-24, 25-29, 30-34, 35-39, 40-44, 45-49, 50-54, 55-59, 60-64, 65-69, 70-74, 75-79, 80-84, 85-89, and $90+$ ) and also by gender and ethnicity strata for specific mental disorders. Because the geographic catchment area providing the majority of SLAM referrals was restricted to southeast London, additional sensitivity analyses were carried out using mortality statistics for London alone in 2008, as published by the Office of National Statistics [16], although, because of restrictions in these source data, wider age strata had to be applied for standardization $(15-24,25-34,35-44,45-54,55-64$, 65-74, 75-84, and 85+).

\section{Results}

A total of 38,066 cases were identified using CRIS with a primary diagnosis, recorded before the end of March 2010, of substance use disorder, schizophrenia, schizoaffective disorder, bipolar affective disorder, depressive episode, or recurrent depressive disorder. Among these, 5,902 cases became inactive to SLAM services before 1 January, 2007 and remained inactive over the followup period and were thus excluded from further analyses. Those younger than 15 years old at the mid-point of 2008 or missing date of birth were also excluded $(\mathrm{n}=$ 445 ). Therefore, a total of 31,719 cases of interest with 1,370 deaths were identified. Of the sample, $1,680(5.3 \%)$ had two diagnoses of interest before the end of 2009, $121(0.4 \%)$ had three diagnoses and six $(0.02 \%)$ had four diagnoses. The most common combinations of diagnoses applied on different occasions in the same individual were schizophrenia and schizoaffective disorders $(n=421)$, followed by substance use disorders and depressive episode or recurrent depressive disorder $(\mathrm{n}=352)$.

Figure 1 summarizes the data retrieval process and number of deaths in each group of interest. Age-standardised mortality ratios (SMRs) of mental disorder diagnoses for the total cohort and stratified by gender are displayed in Table 1 . Mortality was significantly elevated for all disorder groups - highest overall for substance use disorders, followed by schizoaffective disorder, schizophrenia, bipolar affective disorder and depressive episode. The ranking of these disorder-specific SMRs was similar between men and women, except that, in women, there were stronger associations with schizoaffective and bipolar affective disorders compared to schizophrenia. The SMR for any SMI was stronger in men than women, principally because of the marked gender difference for schizophrenia. SMRs for depressive disorders were also higher for men compared to women.

Table 2 displays age- and gender-standardised mortality ratios stratified by ethnic group. SMRs for SMI diagnoses were comparable in size across all groups apart from non-significant findings in the smallest East and

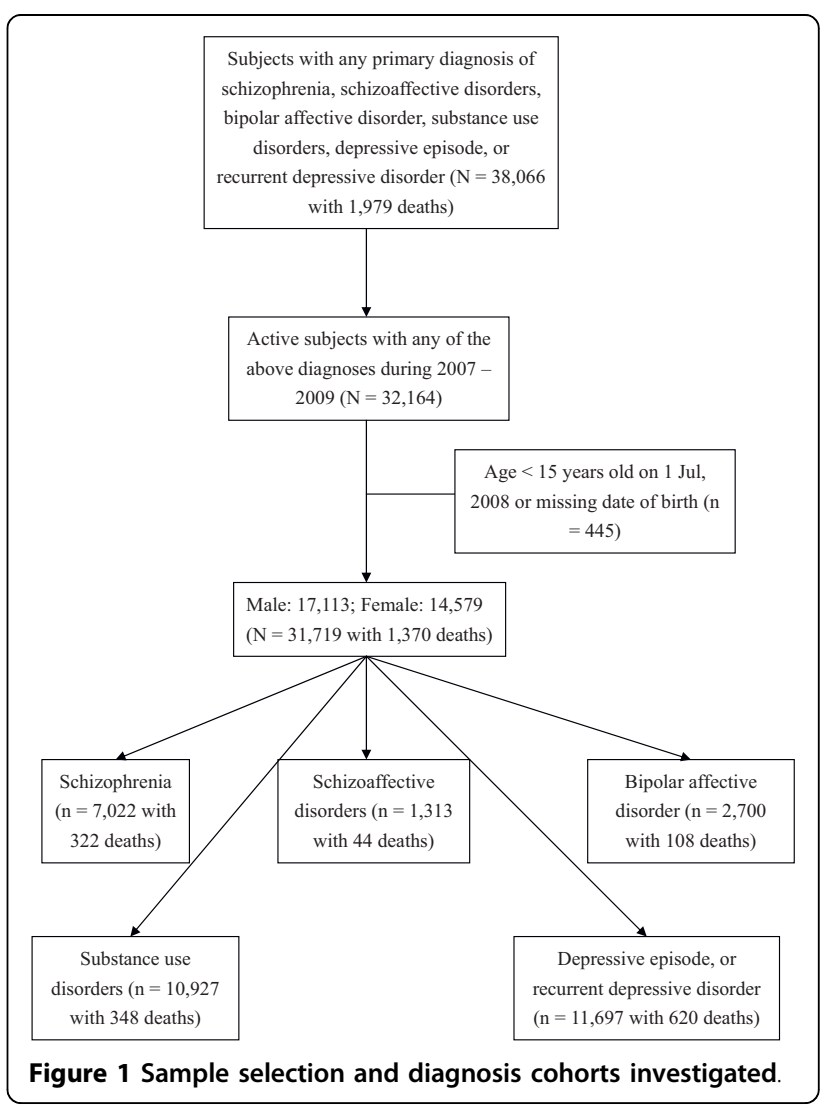


Table 1 Age-standardised mortality ratios (SMRs), stratified by gender, for mental disorder diagnoses in SLAM ${ }^{\#}$

\begin{tabular}{|c|c|c|c|}
\hline \multirow[b]{2}{*}{ Diagnosis } & \multicolumn{3}{|c|}{$\begin{array}{l}\text { Standardised mortality ratio for deaths in 2007-09 } \\
\qquad(95 \% \mathrm{Cl} \text {, number of deaths) }\end{array}$} \\
\hline & Total & Male^ & Female^ $\wedge$ \\
\hline SMI - any & $2.15(1.95-2.36, n=446)^{*}$ & $2.47(2.17-2.80, \mathrm{n}=243)^{*}$ & $1.89(1.64-2.17, n=203)^{*}$ \\
\hline Schizophrenia (F20) & $2.25(2.01-2.51, \mathrm{n}=322)^{*}$ & $2.78(2.40-3.19, \mathrm{n}=196)^{*}$ & $1.74(1.45-2.07, n=126)^{*}$ \\
\hline Schizoaffective disorders (F25) & $2.52(1.83-3.39, n=44)^{*}$ & $2.35(1.35-3.86, n=16)^{*}$ & $2.88(1.91-4.16, \mathrm{n}=28)^{*}$ \\
\hline Bipolar affective disorder (F31) & $1.95(1.60-2.35, n=108)^{*}$ & $1.76(1.27-2.37, \mathrm{n}=43)^{*}$ & $2.21(1.71-2.82, n=65)^{*}$ \\
\hline Substance use disorders (F10 - F19) & $4.17(3.75-4.64, n=348)^{*}$ & $3.60(3.17-4.07, n=254)^{*}$ & $4.67(3.78-5.72, n=94)^{*}$ \\
\hline $\begin{array}{l}\text { Depressive episode and recurrent } \\
\text { depressive disorder (F32 - F33) }\end{array}$ & $1.29(1.19-1.40, n=620)^{*}$ & $1.53(1.36-1.72, n=284)^{*}$ & $1.18(1.06-1.31, \mathrm{n}=336)^{*}$ \\
\hline
\end{tabular}

\# Compared to the population of England and Wales in 2008.

* P-value $<0.05$.

$\wedge$ Standardised by gender- and age-specific mortality rates, separately ( 27 missing values for gender).

South Asian groups. SMRs for substance use disorders appeared more heterogeneous, although confidence intervals were wide and overlapping.

As displayed in Table 3, SMRs for all disorders diminished in strength with increasing age. Even so, all disorders remained significant predictors of mortality in the oldest (65+ years) age stratum.

Secondary sensitivity analyses were carried out by standardising with London mortality in 2008 (details not shown). In summary, SMRs were comparable and, if anything, slightly stronger than those displayed in Tables 1, 2 and 3. For example, the re-calculated SMRs for any SMI were 2.21 for the total sample, 2.49 for men and 1.98 for women. The respective SMRs were $4.28,3.70$ and 4.76 for substance use disorders and 1.42, 1.72 and 1.29 for depressive disorders.

\section{Discussion}

In this analysis, people with mental disorder diagnoses who had had contact with secondary mental healthcare services, had substantially higher mortality than expected in all diagnostic groups examined. The calculated SMRs varied modestly by gender and substantially by age, and also fluctuated markedly across different ethnic groups, which might be caused by small size in specific populations.

People with SMI have substantially higher than expected mortality in all age groups. The raised risk of mortality in these cohorts is consistent with previous studies, indicating that mortality rates among individuals with SMI are higher than that of the general community [6-8]. A previous longitudinal register-based study with the maximum follow up of 18 years in the UK investigated 'any psychiatric diagnosis' as an exposure and found this to be associated with a $65 \%$ higher than expected total mortality [7]. In our analysis, the SMRs of those with SMI suggested a more than two-fold higher mortality, although this may reflect a focus on the more severe mental disorders represented within the SMI label. Causal pathways between mental disorder and mortality have yet to be fully elucidated and are likely to be multiple. While suicide and accidents/violence are important considerations for clinical services, research has tended to show mortality increases across

Table 2 Age- and gender-standardised mortality ratios (SMRs), stratified by ethnicity, for mental disorder diagnoses in SLAM $^{\#}$

\begin{tabular}{|c|c|c|c|}
\hline \multirow[b]{2}{*}{ Ethnic group } & \multicolumn{3}{|c|}{$\begin{array}{l}\text { Standardised mortality ratio for deaths in 2007-09 } \\
\qquad(95 \% \mathrm{Cl} \text {, number of deaths) }\end{array}$} \\
\hline & $\begin{array}{c}\text { SMI } \\
(\mathrm{F} 20,25, \& 31)\end{array}$ & $\begin{array}{l}\text { Substance use disorders } \\
\text { (F10 - F19) }\end{array}$ & $\begin{array}{l}\text { Depressive episode and recurrent depressive disorder } \\
\qquad(\mathrm{F} 32-\mathrm{F} 33)\end{array}$ \\
\hline White British & $1.97(1.72-2.24, n=224)^{*}$ & $3.94(3.47-4.46, n=250)^{*}$ & $1.30(1.18-1.43, n=455)^{*}$ \\
\hline Other white back ground & $2.28(1.74-2.92, n=61)^{*}$ & $4.18(3.08-5.54, n=48)^{*}$ & $1.29(0.99-1.64, \mathrm{n}=63)$ \\
\hline East Asian & $1.63(0.65-3.35, n=7)$ & $0.87(0.02-4.82, n=1)$ & $0.77(0.25-1.81, n=5)$ \\
\hline South Asian & $1.64(0.79-3.02, n=10)$ & $6.55(2.83-12.91, \mathrm{n}=8)^{*}$ & $1.93(1.05-3.23, n=14)^{*}$ \\
\hline $\begin{array}{l}\text { African and other black } \\
\text { background }\end{array}$ & $3.51(2.61-4.62, n=51)^{*}$ & $2.23(1.02-4.23, n=9)^{*}$ & $1.07(0.46-2.11, \mathrm{n}=8)$ \\
\hline Caribbean & $2.06(1.58-2.63, n=64)^{*}$ & $2.73(1.18-5.38, n=8)^{*}$ & $1.52(1.04-2.15, \mathrm{n}=32)^{*}$ \\
\hline Mixed, unknown, and others & $3.21(2.15-4.62, n=29)^{*}$ & $3.76(2.41-5.60, n=24)^{*}$ & $1.39(1.01-1.88, n=43)^{*}$ \\
\hline
\end{tabular}

\# Compared to the population of England and Wales in 2008.

* P-value $<0.05$ 
Table 3 Age-standardised mortality ratios (SMRs), stratified by age group, for mental disorder diagnoses in SLAM $^{\#}$

\begin{tabular}{|c|c|c|c|}
\hline \multirow[b]{2}{*}{ Diagnosis } & \multicolumn{3}{|c|}{$\begin{array}{l}\text { Standardised mortality ratio for deaths in 2007-09 } \\
\qquad(95 \% \mathrm{Cl} \text {, number of deaths) }\end{array}$} \\
\hline & 15 - 44 years old & 45 - 64 years old & $65+$ years old \\
\hline SMI - any & $4.47(3.49-5.64, n=71)^{*}$ & $3.10(2.61-3.66, n=140)^{*}$ & $1.60(1.40-1.82, n=235)^{*}$ \\
\hline Schizophrenia (F20) & $4.73(3.52-6.22, n=51)^{*}$ & $3.44(2.82-4.16, n=106)^{*}$ & $1.63(1.39-1.89, n=165)^{*}$ \\
\hline Schizoaffective disorders (F25) & $3.96(1.81-7.52, \mathrm{n}=9)^{*}$ & $2.71(1.48-4.55, n=14)^{*}$ & $2.10(1.30-3.21, \mathrm{n}=21)^{*}$ \\
\hline Bipolar affective disorder (F31) & $4.09(2.38-6.54, n=17)^{*}$ & $2.58(1.77-3.64, n=32)^{*}$ & $1.51(1.15-1.95, \mathrm{n}=59)^{*}$ \\
\hline Substance use disorders (F10 - F19) & $6.81(5.77-7.98, n=153)^{*}$ & $4.40(3.70-5.20, n=139)^{*}$ & $1.91(1.44-2.48, n=56)^{*}$ \\
\hline $\begin{array}{l}\text { Depressive episode and recurrent } \\
\text { depressive disorder (F32 - F33) }\end{array}$ & $3.21(2.40-4.20, n=53)^{*}$ & $1.75(1.35-2.22, \mathrm{n}=66)^{*}$ & $1.18(1.08-1.28, \mathrm{n}=501)^{*}$ \\
\hline
\end{tabular}

\# Compared to the population of England and Wales in 2008.

* P-value $<0.05$

all major causes, including cardiovascular diseases (heart attack and stroke) [11]. These may be influenced by direct effects of mental disorder symptoms (for example on suicide and accidents). Pathways may also reflect adverse lifestyle factors influenced by the presence of mental disorders and themselves responsible for associations with mortality; these include worse nutrition, physical inactivity, alcohol use, smoking, and illicit drug use [2-4]. Adverse effects from psychotropic (particularly antipsychotic) medication have also received increasing consideration in terms of their role in raised mortality as an outcome [17]. Specifically, antipsychotic agents are often prescribed long term and may increase the risks of diabetes mellitus and cardiovascular diseases with events including QT-interval prolongation, ventricular arrhythmias, pulmonary embolus, atherosclerosis, and sudden cardiac death $[4,18-20]$. The analyses presented here were not intended to elucidate causal pathways, but rather to constitute the first stage in a series of investigations of these issues with future studies clarifying further the role of socioeconomic status, education and cognitive abilities which are all associated with higher mortality and might confound the reported associations [21,22].

Higher mortality in people with schizophrenia has been recognised since the $1930 \mathrm{~s}$ [11]. Nonetheless, as stated earlier, this mortality gap has remained stubbornly unchanged [10], and may even have increased over time $[5,11]$, despite developments in mental health services and the introduction of better tolerated antipsychotic agents. Previous studies suggest that around two-thirds of the excess mortality among people with schizophrenia may be attributable to natural causes, as discussed above [23], with the remaining one third being due to suicide and other unnatural causes [23-26]. Regarding bipolar disorder, a non-significant raised SMR for all causes of death was reported in another study performed in South London with a more limited sample size (239 cases with 42 deaths over 19 years) [27]. Regarding SMRs for cases with depressive episode or recurrent depressive disorder, a particularly high mortality risk was identified among the younger age stratum (15-44 year olds, Table 3). However, the SMRs for depression were relatively low [28]. This finding may be due to the fact that people with depression known to secondary care are not representative of those with the disorder in the community and, in particular, that referral bias for secondary care favours those with relatively good health or higher social class $[29,30]$.

Our findings are, we believe, novel in the presentation of SMRs stratified by age, gender, and ethnicity. Effect modification, where demonstrated, may provide at least some supportive evidence regarding causal pathways since it indicates uneven distribution of risk; however, such conclusions can only be drawn tentatively and require confirmatory investigation. Gender differences in the associations of interest might reflect different levels of environmental support (for example, lower social support for men with schizophrenia might account for the higher SMR in that group), or might reflect gender differences in the severity of the condition in question or in the level of comorbidity with other physical, mental or personality disorder [31]. The substantial differences in SMRs between ethnic groups again suggest that some of the associations between SMI and mortality may be socially mediated, although confidence intervals were wide for many groups and negative findings should be viewed with caution. The diminution of mortality risk with age may reflect survival effects, with those surviving with serious mental disorder to age $65+$ being relatively healthier in other respects. Alternatively, people with SMI in older age ranges may be more likely to remain in contact with mental health services and adhere to treatment, whether for mental or physical disorders. However, the age diminution could also reflect differences in the nature of the underlying disorders, such as symptomatic differences between early- and late-onset schizophrenia, or differences in substances misused in younger and older adults. Further, it may reflect the excess risk of SMI being additive rather than 
multiplicative, so it is obscured by deaths from other causes in older age groups $[7,20]$.

This investigation has a number of strengths. We were able to draw on a large numbers of case records from the largest single provider of secondary mental healthcare in Europe. The National Health Service presents additional contextual advantages because of its near-total coverage of all aspects of healthcare in the UK. This investigation was able to draw on complete electronic clinical records of more than 31,000 patients diagnosed with SMI, depression or substance use disorders providing the statistical power to be able to differentiate between disorders as well as explore subgroup-specific mortality risk.

However, potential limitations should also be considered. First of all, confounders other than age and gender might still exist. Case registers derived from secondary healthcare offer particular advantages for investigating "high penetrance" disorders - i.e. those like schizophrenia and bipolar affective disorder where the chances of secondary care contact are high. For lower penetrance disorders, inferences need to be more cautious and both depressive and substance use disorders fall into this category - i.e. cases known to secondary care may reflect more severe primary disorders, comorbidity, environmental disadvantage or referral biases. Prevalence bias is an additional issue which needs consideration, in that the cases known to a service within a given time period are likely to be dominated by those with long and relapsing clinical courses - they therefore cannot be taken to generalise to incident cases. A further methodological challenge was that service data were principally (but not entirely) derived from a single catchment area whereas expected deaths were derived from national data. However, a sensitivity analysis using London-specific data, described above, did not reveal any meaningful differences. Finally, we have not included data on causes of death and further research is required for this, as well as for investigating specific risk factors for mortality within particular disorders. Diagnostic categories overlapped since a proportion of individuals suffer from more than one mental disorder, but no attempt in this analysis was made to consider comorbidity.

\section{Conclusions}

People with serious mental illness, substance use disorders and depressive disorders continue to be at higher risk of mortality compared to the general population. Furthermore, mortality risk differs substantially with age, diagnosis, gender and ethnicity. Further research into specific risk groups is required.

\section{Acknowledgements}

The development of the SLAM BRC Case Register has been funded by two Capital Awards from the UK National Institute for Health Research and is further supported through the BRC Nucleus funded jointly by the Guy's and St Thomas' Trustees and South London and Maudsley Special Trustees. CKC, $\mathrm{RH}, \mathrm{AF}, \mathrm{MB}, \mathrm{MH}$ and $\mathrm{RS}$ are funded by the National Institute for Health Research (NIHR) Specialist Biomedical Research Centre for Mental Health at the South London and Maudsley NHS Foundation Trust and Institute of Psychiatry, King's College London. WL is funded by the UK Medical Research Council.

\section{Author details}

${ }^{1}$ King's College London, Section of Epidemiology, Dept of Health Service and Population Research, Institute of Psychiatry, London, UK. 'King's College London, Academic Dept Psychological Medicine, Institute of Psychiatry, London, UK.

\section{Authors' contributions}

All the authors listed contributed themselves in the process of hypothesis generation, data collection, statistical analyses, or manuscript preparation, and fulfilled the criteria for authorship. CKC and $\mathrm{RH}$ carried out the data retrieval, statistical analyses, and manuscript drafting. $A F, M B, W L$ and $R S$ participated in the hypothesis generation, data management, and assistant on manuscript preparation. $\mathrm{CKC}, \mathrm{RH}, \mathrm{MH}$ and $\mathrm{RS}$ conceived of the study, participated in its design, and implemented the project. All the authors read and approved the final manuscript.

\section{Competing interests}

The authors declare that they have no competing interests.

Received: 23 June 2010 Accepted: 30 September 2010 Published: 30 September 2010

\section{References}

1. Auquier $P$, Lancon C, Rouillon F, Lader M, Holmes C: Mortality in schizophrenia. Pharmacoepidemiol Drug Saf 2006, 15:873-879.

2. Robson D, Gray R: Serious mental illness and physical health problems: a discussion paper. Int J Nurs Stud 2007, 44:457-466.

3. Fagiolini A, Goracci A: The effects of undertreated chronic medical illnesses in patients with severe mental disorders. J Clin Psychiatry 2009, 70(Suppl 3):22-29.

4. Brown S, Birtwistle J, Roe L, Thompson C: The unhealthy lifestyle of people with schizophrenia. Psychol Med 1999, 29:697-701.

5. Osby U, Correia N, Brandt L, Ekbom A, Sparen P: Time trends in schizophrenia mortality in Stockholm county, Sweden: cohort study. BMJ 2000, 321:483-484

6. Colton CW, Manderscheid RW: Congruencies in increased mortality rates, years of potential life lost, and causes of death among public mental health clients in eight states. Prev Chronic Dis 2006, 3:A42.

7. Baxter DN: The mortality experience of individuals on the Salford Psychiatric Case Register. I. All-cause mortality. Br J Psychiatry 1996, 168:772-779.

8. Osborn DP, Levy G, Nazareth I, Petersen I, Islam A, King MB: Relative risk of cardiovascular and cancer mortality in people with severe mental illness from the United Kingdom's General Practice Rsearch Database. Arch Gen Psychiatry 2007, 64:242-249.

9. Osborn D, Levy G, Nazareth I, King M: Suicide and severe mental illnesses. Cohort study within the UK general practice research database. Schizophr Res 2008, 99:134-138.

10. Tiihonen J, Lonnqvist J, Wahlbeck K, Klaukka T, Niskanen L, Tanskanen A Haukka J: 11-year follow-up of mortality in patients with schizophrenia: a population-based cohort study (FIN11 study). Lancet 2009, 374:620-627.

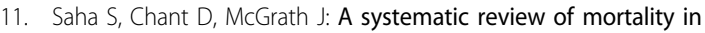
schizophrenia: is the differential mortality gap worsening over time? Arch Gen Psychiatry 2007, 64:1123-1131.

12. McGirr A, Turecki G: What is specific to suicide in schizophrenia disorder? Demographic, clinical and behavioural dimensions. Schizophr Res 2008, 98:217-224.

13. Ciranni MA, Kearney $T E$, Olson KR: Comparing acute toxicity of first- and second-generation antipsychotic drugs: a 10-year, retrospective cohort study. J Clin Psychiatry 2009, 70:122-129.

14. Stewart R, Soremekun M, Perera G, Broadbent M, Callard F, Denis M, Hotopf M, Thornicroft G, Lovestone S: The South London and Maudsley NHS Foundation Trust Biomedical Research Centre (SLAM 
BRC) case register: development and descriptive data. BMC Psychiatry 2009, 9:51.

15. Keown P, Mercer G, Scott J: Retrospective analysis of hospital episode statistics, involuntary admissions under the Mental Health Act 1983, and number of psychiatric beds in England 1996-2006. BMJ 2008, 337:a1837.

16. Mortality statistics, Deaths registered in 2008, UK. [http://www.statistics. gov.uk/downloads/theme_health/DR2008/DR_08.pdf].

17. Weinmann $\mathrm{S}$, Read J, Aderhold V: Influence of antipsychotics on mortality in schizophrenia: systematic review. Schizophr Res 2009, 113:1-11.

18. Davidson M: Risk of cardiovascular disease and sudden death in schizophrenia. J Clin Psychiatry 2002, 63(Suppl 9):5-11.

19. Koponen H, Alaraisanen A, Saari K, Pelkonen O, Huikuri H, Raatikainen MJ, Savolainen M, Isohanni M: Schizophrenia and sudden cardiac death: a review. Nord J Psychiatry 2008, 62:342-345.

20. Sicouri S, Antzelevitch C: Sudden cardiac death secondary to antidepressant and antipsychotic drugs. Expert Opin Drug Saf 2008, 7:181-194.

21. Kuh $D$, Hardy R, Langenberg C, Richards M, Wadsworth ME: Mortality in adults aged 26-54 years related to socioeconomic conditions in childhood and adulthood: post war birth cohort study. BMJ 2002, 325:1076-1080.

22. Kuh D, Richards M, Hardy R, Butterworth S, Wadsworth ME: Childhood cognitive ability and deaths up until middle age: a post-war birth cohort study. Int J Epidemiol 2004, 33:408-413.

23. Brown S: Excess mortality of schizophrenia. A meta-analysis. $\mathrm{Br} J$ Psychiatry 1997, 171:502-508.

24. Hawton K, Sutton L, Haw C, Sinclair J, Deeks JJ: Schizophrenia and suicide: systematic review of risk factors. Br J Psychiatry 2005, 187:9-20.

25. Haukka J, Tiihonen J, Harkanen T, Lonnqvist J: Association between medication and risk of suicide, attempted suicide and death in nationwide cohort of suicidal patients with schizophrenia. Pharmacoepidemiol Drug Saf 2008, 17:686-696.

26. Ward A, Ishak K, Proskorovsky I, Caro J: Compliance with refilling prescriptions for atypical antipsychotic agents and its association with the risks for hospitalization, suicide, and death in patients with schizophrenia in Quebec and Saskatchewan: a retrospective database study. Clin Ther 2006, 28:1912-1921.

27. Dutta R, Boydell J, Kennedy N, Van Os J, Fearon P, Murray RM: Suicide and other causes of mortality in bipolar disorder: a longitudinal study. Psychol Med 2007, 37:839-847.

28. Wulsin $L R$, Vaillant $G E$, Wells VE: A systematic review of the mortality of depression. Psychosom Med 1999, 61:6-17.

29. Telford R, Hutchinson A, Jones R, Rix S, Howe A: Obstacles to effective treatment of depression: a general practice perspective. Fam Pract 2002, 19:45-52.

30. Cape J, Parham A: Rated casemix of general practitioner referrals to practice counsellors and clinical psychologists: a retrospective survey of a year's caseload. Br J Med Psychol 2001, 74(Pt 2):237-246.

31. McCormick B, Blum N, Hansel R, Franklin JA, St John D, Pfohl B, Allen J, Black DW: Relationship of sex to symptom severity, psychiatric comorbidity, and health care utilization in 163 subjects with borderline personality disorder. Compr Psychiatry 2007, 48:406-412.

\section{Pre-publication history}

The pre-publication history for this paper can be accessed here: http://www.biomedcentral.com/1471-244X/10/77/prepub

doi:10.1186/1471-244X-10-77

Cite this article as: Chang et al: All-cause mortality among people with serious mental illness (SMI), substance use disorders, and depressive disorders in southeast London: a cohort study. BMC Psychiatry 2010 10:77.

\section{Submit your next manuscript to BioMed Central and take full advantage of:}

- Convenient online submission

- Thorough peer review

- No space constraints or color figure charges

- Immediate publication on acceptance

- Inclusion in PubMed, CAS, Scopus and Google Scholar

- Research which is freely available for redistribution

Submit your manuscript at www.biomedcentral.com/submit
Biomed Central 